\title{
TEJIENDO ENTRE REDES DIVERSAS: REFLEXIONES A PARTIR DE UNA ETNOGRAFÍA MULTISITUADA CON LOS PUEBLOS INDÍGENAS DE LA SIERRA NEVADA DE SANTA MARTA, CARIBE COLOMBIANO
}

ANA MILENA HORTA PRIETO ${ }^{1}$

UFRGS

\begin{abstract}
RESUMEN: La Sierra Nevada de Santa Marta, Colombia, es territorio de 4 pueblos indígenas: Kogui, Wiwa, Arhuaco y Kankuamo. Estos comparten la Ley de Sé, orden natural del universo, que en este trabajo se aborda como cosmopolitica (Stengers), a partir de la cual se gestiona la alteridad y se define la función de todos los seres existentes, desde una ontología animista (Descola). Los trabajos espirituales, entre los cuales se cuentan los pagamentos, son rituales en los que se realiza el tejido vital entre entidades, a partir del cual pueden circular los flujos energéticos y de pensamiento, que relacionan mamos (autoridades tradicionales), dueños de los existentes (Jaba y jate), y materiales sagrados, en una relación de intercambio que permite que las partes se reconfiguren y se apropien del sewá (conocimiento) que cada uno posee. Algunos de estos materiales han sido removidos del territorio, frente a lo cual las organizaciones indígenas han comenzado un proceso de patrimonialización como estrategia de protección, interactuando con entidades del estado que tienen influencia en la configuración del mismo desde su dominio. En este marco, a partir de la categoría de cosmopolitica y del entendimiento del conocimiento indígena como epistemología, este trabajo reflexiona sobre el posicionamiento del antropólogalo en ese ordenamiento desde donde se puede tejer entre redes de ontologías diferentes.
\end{abstract}

PALABRAS ClAVE: Sierra Nevada de Santa Marta; Cosmopolitica; Patrimonio.

\begin{abstract}
The Sierra Nevada of Santa Marta, Colombia, is the territory of four indigenous groups: Kogui, Wiwa, Arhuaco and Kankuamo. They share the ley de Sé, the natural order of the universe, addressed in this article as their cosmopolitics (Stengers), which manages alterity defining the function of all the existent, from an animistic ontology (Descola). The trabajos espirituales, spiritual works, including the pagamentos, are rituals for the vital woven between entities which allows the energetic and though flows that connects mamos (traditional spiritual authorities), owners of beings (jaba and jate), and the sacred materials, in an exchange relation, enabling these parts to reconfigure and appropriate each others sewá (knowledge). Because some of these materials have been removed from their original territory, the indigenous organizations and some concerned state entities have started a process for the

\footnotetext{
${ }^{1}$ Ana Milena Horta é formada em Antropologia pela Universidad de Los Andes, Colômbia. Atualmente realiza o mestrado em Antropologia social na Universidade Federal de Rio Grande do Sul, sob orientação de Sergio Baptista da Silva. Tabalhou em projetos de participação social e meio ambiente com diferentes povos indígenas das regiões da Amazonia, da orinoquia e do caribe colombiano, entre eles os Kogui e os Arhuaco. E-mail: anamilenahorta@yahoo.es.
}

Espaço Ameríndio, Porto Alegre, v. 8, n. 1, p. 135-161, jan./jun. 2014. 
ANA MILENA HORTA PRIETO - Tejiendo entre redes diversas

nomination of their sacred materials as heritage as a protective strategy. Within this general context, and from the notion of cosmopolitics and the understanding of the indigenous knowledge as epistemology, this article reflects on the ideological stance of the anthropologist regarding the management from where different nets with different ontologies can be woven.

KEYWORDS: Sierra Nevada de santa Marta; Cosmopolitics; Heritage.

\section{Introducción}

La Sierra Nevada de Santa Marta (SNSM), ubicada al norte de Colombia en la región Caribe, está poblada actualmente por cuatro pueblos indígenas que comparten un mismo origen mítico, y que se declaran descendientes del pueblo Tayrona, constructores de lo que ahora es una de las zonas arqueológicas más importantes del país. Estos cuatro pueblos son los Kogui, Wiwa, Arhuacos y Kankuamos. En su territorio se sobreponen figuras territoriales diversas tales como la de territorio ancestral, Resguardo ${ }^{2}$, Parque Arqueológico Teyuna Ciudad Perdida, Reserva de Biosfera, Parque Nacional Natural Sierra Nevada de Santa Marta, lo que da cuenta de la cantidad de intereses y perspectivas que se han tejido históricamente en el área.

Estos intereses, de la mano de una serie de procesos nacionales y de la región ${ }^{3}$, han influenciado fuertemente las condiciones de vida de estos pueblos y han motivado un fuerte proceso organizativo por el reconocimiento de sus derechos colectivos como pueblo, la consolidación del territorio y su autonomía. El contexto global de políticas multiculturales y crisis ambiental, ha impulsado su movimiento y su interacción con redes de representaciones, políticas y discursos científicos, facilitando tanto la configuración de su identidad, que está

\footnotetext{
${ }^{2}$ La figura de Resguardo, de acuerdo a la legislación colombiana, se refiere a territorios de propiedad colectiva indígena, reconocidos por el estado, cuya administración y manejo se realiza de acuerdo a los parámetros culturales y costumbres propias de los pueblos que los habitan. Se reconocen las autoridades propias como autoridad pública especial indígena. El territorio ancestral, por el contrario, es un reconocimiento cultural, pero no otorga los derechos que la figura del resguardo si da. El área cuenta con tres resguardos reconocidos: Kogui malayo arhuaco, Arhuaco y Kankuamo.

3 Misiones capuchinas, colonización campesina, cultivos ilícitos, conflicto nacional guerrillaparamilitarismo- ejército, desplazamiento forzado, intereses económicos (mineros, turísticos, proyectos desarrollistas) y ambientales (declaración de áreas de conservación, estrategias regionales de conservación ambiental y cultural).
}

Espaço Ameríndio, Porto Alegre, v. 8, n. 1, p. 135-161, jan./jun. 2014. 
asociada a tradición, ecología y espiritualidad (URIBE, 1988; ORRANTIA, 2002), como al desarrollo de estrategias y articulaciones para el reconocimiento de sus derechos en términos, prácticas y discursos que cada vez les son menos ajenos.

En este marco, estos pueblos indígenas han creado asociaciones ${ }^{4}$ para responder a las exigencias para dialogar con entidades estatales nacionales, internacionales y ONG's para luchar por su territorio y autonomía y defender lo que ellos llaman su tradición. Después de varios años en este proceso, que implicó el ajuste y acoplamiento de las nuevas autoridades -las exigidas por la ley con la creación de los resguardos y los líderes de las organizaciones- a las autoridades tradicionales, se puede decir que han logrado ordenarse para interlocutar con entidades diversas que tienen injerencia en su territorio, intentando responder a lo que los indígenas reconocen como "orden natural de la Sierra Nevada".

Sahlins (1997a y 1997b) afirma que desde una perspectiva culturalista nostálgica, estos procesos de encuentro pueden verse como parte de una globalización homogenizante, pero argumenta que es más interesante entender las rupturas, continuidades, apropiaciones y reinterpretaciones que se dan en estas dinámicas de contacto. En otro texto (SAHLINS, 2001), responde a la crítica de la instrumentalización de la cultura, según la cual esta puede responder a intereses de elites dominantes, afirmando que siempre ha respondido a exigencias diversas, bien sea en sus configuraciones internas o externas, e invita a ir más allá de juicios morales para entender cómo es que se relacionan esas estrategias e intereses.

Estas dinámicas de contacto permiten reconfiguraciones en doble vía, y si bien están enmarcadas en relaciones de poder, este puede es reconfigurado y transforma sus mecanismos de captura, a la vez que aquellos considerados subalternos también se reconfiguran intentado

\footnotetext{
${ }^{4}$ Las organizaciones indígenas actuales son Organización Wiwa Yugumain Bunkwanarrwa Tayrona, OWYBT; Organización Indígena Kankuama, OIK; Organización Gonawindua Tayrona, OGT; Confederación Indígena Tayrona, CIT; Concejo Territorial de Cabildos, CTC. Es decir que una por cada pueblo y otra conjunta, tras una historia de agrupaciones y desagrupaciones. Adicional a esto, la figura de resguardo establece que debe haber un representante de la comunidad que ejerza un liderazgo y con quien se pueda interlocutar directamente, un cabildo. Para el caso de la Sierra, esto fue traumático ya que su sistema de autoridades no es jerárquico y tampoco centralizado. Para profundizar en estos aspectos organizativos y de interlocución con el Estado, ver Ulloa (2004).
}

Espaço Ameríndio, Porto Alegre, v. 8, n. 1, p. 135-161, jan./jun. 2014. 
escapar a la captura y reivindicando su visión del mundo, su mundo.

En este marco, este trabajo aborda un caso en el que se articulan nociones diversas sobre los objetos sagrados de los pueblos indígenas de la Sierra Nevada de Santa Marta. El discurso del patrimonio en la década de 1970 permitió la extracción de dichos objetos, su traslado a museos y la constitución del Parque Arqueológico Teyuna, uno de los más importantes del país, declarando su pertenencia a "todos los colombianos". Actualmente las organizaciones indígenas, por medio también del discurso de patrimonio y sus procesos actuales buscan la protección y la elaboración de un plan de salvaguardia de los objetos y los sitios sagrados como estrategia de protección, con la condición de que se maneje su condición integral que no se enmarca en las distinciones de cultura material e inmaterial. Es un proceso que apenas comienza y que plantea muchos retos para lograr las expectativas de las partes. Este trabajo ${ }^{5}$ busca aportar a esta articulación del mundo Kogui con un discurso perteneciente a una epistemología diferente, a partir del entendimiento de aspectos centrales de su cosmopolitica (STENGERS, 2003), la reivindación del pensamiento Kogui como epistemología, y algunas reflexiones sobre cómo ha establecido una relación entre epistemologías diferentes a partir de los objetos sagrados. En este marco hago referencia a la función del quehacer antropológico en el orden del mundo Kogui y este caso de interrelación.

\section{Cosmopolitica kogui}

El universo Kogui se origina en Aluna. Aluna es el espacio-tiempo del origen, cuando todo era pensamiento y espíritu, cuando todo era oscuridad y solo existía la madre. Poco a poco, la madre fue creando mundos, y con ellos padres y madres (jate y jaba), que dieron origen a otros mundos y seres que solo existían en pensamiento. Esta creciente familia de padres ancestrales creó 9 mundos en total, todos existían en pensamiento. Estos mundos son planos que conforman un huevo, en

\footnotetext{
${ }^{5} \mathrm{Si}$ bien este es un proceso de los 4 pueblos de la Sierra, este trabajo se refiere a nociones principalmente del pueblo Kogui.

${ }^{6}$ Palabras en el lenguaje Kogui para padre y madre.
}

Espaço Ameríndio, Porto Alegre, v. 8, n. 1, p. 135-161, jan./jun. 2014. 
cuya mitad está el mundo que habitamos. Este huevo es entendido como el útero materno, pero también se interpreta como un ovillo ${ }^{7}$ atravesado por un huso ${ }^{8}$ que es sostenido por cuatro padres (REICHEL, 1985). Los padres y madres de las familias ancestrales dieron origen a todo lo que existe: animales, lagunas, ríos, frutas, gente, plantas, piedras, sentimientos, etc. A medida que lo iban creando, se iba determinando su función en el mundo y su relación con los otros seres. Seránkua, hijo de la madre, fue el padre creador que materializó todo lo que ya existía en la oscuridad en espíritu y pensamiento. El pensamiento materializado es el territorio ancestral, Sé nenulang, la Sierra Nevada de Santa Marta. Así fue que al materializarse el mundo "amaneció", y los mandatos de la madre, los códigos según los cuales los seres deben relacionarse y la función que cada uno debe cumplir para mantener el orden y el equilibro, fueron inscritos en el territorio y constituyen la ley de origen o la Ley de Sé. Esta ley determina la manera como el territorio debe ser repartido entre los pueblos a los que le fue entregado, los Kogui, Wiwa, Arhuacos y Kankuamos. Así mismo, la Ley de Sé expresa que el territorio es el cuerpo vivo de la madre, de manera que indica los cuidados y el manejo que se le debe dar a Sé nenulang para que ese cuerpo esté libre de enfermedades, y se mantenga el equilibrio del origen, que permite la existencia de todos los seres que lo componen.

Este equilibrio se mantiene cuando todo lo que existe cumple con la función que le corresponde, se cumplen los mandatos de comportamiento y las prácticas que permiten que los padres y madres (jate y jaba) mantengan un flujo de relaciones entre sí, conformando un tejido a partir de las conexiones que permiten la integralidad y la vida de la Sierra (OGT, 2012). Los jaba y jate son los órganos del cuerpo de la Sierra, son los nudos del tejido que permite la vitalidad del cuerpo. Esta idea del territorio como tejido es central en el mundo de los indígenas de la Sierra. El territorio es un tejido de relaciones entre seres humanos y no humanos que cumpliendo la misión para la cual fueron creados, permiten el flujo de pensamiento y energía que hacen del territorio un cuerpo vivo y sano. Son las conexiones y el cumplimiento de la misión de cada ser, lo que permite el equilibrio. Así como el

\footnotetext{
${ }^{7}$ Bola que se forma al hilar algodón.

${ }^{8}$ Instrumento usado para hilar algodón.
}

Espaço Ameríndio, Porto Alegre, v. 8, n. 1, p. 135-161, jan./jun. 2014. 
universo es un ovillo de hilo, la existencia y la vida son posibles tejiendo, relacionando seres que cumplen una función para mantener un cuerpo vivo. Dicen los mitos arhuacos, que Seránkua, el padre de la creación, materializó el mundo tejiendo un hilo de pensamiento en forma de espiral a través de los picos nevados (GIRALDO, 2010), que son grandes jefes del territorio.

Los Jaba y Jate, madres y padres, son jefes, los dueños espirituales de todo lo que existe, están inscritos en el territorio y en ellos se contienen los principios de la Ley de Sé. Se trata de entidades vivas que deben ser alimentadas y cuidadas para que no se enfermen y puedan cumplir con su función, ya sea regular, vigilar o controlar. Son el origen de animales, fenómenos naturales, enfermedades, sentimientos, así como principios del gobierno, la autoridad, y la sociabilidad. Los jaba y jate, son los encargados de regular, controlar y hacer que cada existente cumpla con su función. Por esto, se les debe pedir permiso, pagar y agradecer el uso y la regulación que ejercen sobre lo que les corresponde. Sin embargo, no pueden cumplir su función en aislamiento, por lo que deben estar en conexión con otras entidades. Esta conexión es posible mediante flujos naturales como los recorridos del agua desde las cumbres hasta el mar, como mediante los Pagamentos, que hacen parte de una serie de rituales que los Kogui llaman trabajos espirituales o trabajos tradicionales ${ }^{9}$.

Los mamos, son las autoridades espirituales que mediante una educación especial que reciben desde niños y el seguimiento de determinadas normas de comportamiento, que buscan purificar su espíritu y pensamiento, pueden acceder a aluna ${ }^{10}$, que también es un estado del pensamiento y el espíritu, en el que pueden comunicarse con los jaba y jate. Así, aluna no es un tiempo pasado del origen, permanece en el presente; no es solamente una referencia al tiempo mítico, sino que es parte del presente y de un mundo donde lo material y lo espiritual no son campos separados y mucho menos opuestos.

En aluna los mamos reciben sewá, que es un conocimiento

\footnotetext{
${ }^{9}$ Los trabajos tradicionales incluyen también otros rituales como la consulta, que es cuando el mamo entra en contacto con los jaba y jate para decidir qué hacer en determinada situación; confesión, que se realiza cuando alguna persona quiere consultar algo al mamo (BOCAREJO, 2002), así como cuando se va a ir a algún lugar sagrado y deben "limpiarse" los sentimientos y las intenciones.

${ }^{10}$ Para ampliar la información sobre la formación de los mamos, ver Reichel (1976).
}

Espaço Ameríndio, Porto Alegre, v. 8, n. 1, p. 135-161, jan./jun. 2014. 
específico que determina la función de cada uno. Esta noción de sewá es muy importante y tiene varias implicaciones. Es un conocimiento que otorga la capacidad de actuar en el mundo, y define la función que se tiene en este, a partir de un dominio específico. Sin embargo, este dominio y esta función no son terrenos fijos y predeterminados, pueden sobreponerse y variar dependiendo de las relaciones entre los seres. Los jaba y jate tienen sewá. Todo hombre tiene un sewá, que recibe cuando de niño pasa a ser hombre y recibe su poporo ${ }^{11}$ para mascar coca. Los mamos en su proceso de formación y conocimiento van adquiriendo sewás.

En aluna al comunicarse con los jaba y jate, los mamos pueden leer los códigos de la ley de Sé. Son los encargados de realizar los pagamentos, que son las entregas de "materiales" u "objetos" sagrados a los jaba y jate, como pago o alimento a cambio de usufructuar lo que a esa jaba o jate le pertenece. Los pagamentos permiten mantener las conexiones y el tejido de la vida entre los dueños espirituales y los habitantes del mundo. El alimento espiritual consiste en mantener ese flujo vital que circula entre las entidades. Esta red de conexiones "hace circular la energía, la fuerza que da la vida de todos los elementos de la Sierra Nevada" (OGT, 2012, p. 17).

La centralidad de los pagamentos y las relaciones con otras entidades del mundo ha sido resaltada en las descripciones $\mathrm{e}$ investigaciones de los indígenas de la Sierra Nevada. Desde los primeros acercamientos etnográficos de Reichel Dolmatoff (1985) con los Kogui en la década de 1940, es claro que el universo está compuesto por seres que conforman una gran familia que proviene de la madre universal, que todo lo que existe es gente también en Aluna, y que por tanto deben realizar también las prácticas y rituales sociales que mantienen las conexiones de las que depende la integralidad y la vida.

Sin embargo, la interpretación de Reichel tiene el inconveniente de abordar esta concepción como una construcción simbólica sobre la naturaleza, enfoque clave en la antropología de la época, con bases epistemológicas de la racionalidad moderna.

Autores como Descola (2001 y 2012), Latour (2012), Viveiros de

\footnotetext{
${ }^{11}$ El poporo es un recipiente de calabazo en el que se guarda cal, resultante de la trituración de conchas de mar, que se mezcla con saliva para masticar coca.
}

Espaço Ameríndio, Porto Alegre, v. 8, n. 1, p. 135-161, jan./jun. 2014. 
Castro (2011), Stengers (2003), Ingold (2012), entre otros, han señalado las implicaciones de la separación entre naturaleza y cultura como base del desarrollo del pensamiento científico.

De acuerdo a Ingold (2001), el pensamiento científico arraigó la idea de que el mundo es un externo real, objetivo, al cual los humanos le otorgan sentidos a partir de valores o nociones culturales construidas. Así, se entiende que hay un conocimiento objetivo capaz de abordar la realidad de esa exterioridad llamada mundo, que son las ciencias naturales. Por otro lado, hay otros tipos de pensamientos (no conocimientos legítimos) que son intentos de comprender el mundo. Estos son aproximaciones que bajo el rotulo de culturales, se entienden como representaciones, construcciones cognitivas internas del mundo exterior. Una de las consecuencias de esta separación, es que estas construcciones culturales son entendidas como visiones folk del mundo, perspectivas ingenuas que no logran acceder al mundo real al que si accede la epistemología occidental euro-centrada, gracias al pensamiento científico. En este marco, Reichel, interpreta la visión Kogui del universo como una construcción intelectual compleja, pero que constituye una proyección que no logra acceder a la realidad del mundo12; en estos términos se trata de una relación simbólica, representativa, con la exterioridad naturaleza, no de una alteridad con otros existentes ubicados en el mismo plano del ser. Así mismo concordando con la racionalidad moderna, no le da el carácter de actor a las otras entidades que conforman el mundo (piedras, objetos, etc.) con base en la separación sujeto- objeto, dualidad consecuente en este marco de pensamiento, donde el objeto es inerte y no tiene agencia; esto limita la perspectiva de análisis al abordar la cuestión de los pagamentos, que se desarrollará más adelante.

Estas nociones occidentales fortalecidas no solo desde la antropología sino también desde diversas redes socio técnicas relacionadas con políticas de conservación y multiculturales, han aportado a la construcción de una imagen de los pueblos de la Sierra Nevada, principalmente de los Kogui -de quienes se considera que conservan con mayor recelo el conocimiento ancestral- asociada a

\footnotetext{
${ }^{12}$ Vale la pena resaltar, que el trabajo de Reichel Dolmatoff, fue de suma importancia tanto para el conocimiento y valoración de la Sierra Nevada, como para el desarrollo de la antropología en Colombia.
}

Espaço Ameríndio, Porto Alegre, v. 8, n. 1, p. 135-161, jan./jun. 2014. 
tradición, conservación ambiental y un alto grado de espiritualidad (URIBE, 1988; ULLOA, 2004; ORRANTIA, 2002), que ha llevado tanto a su respeto y valoración, como a su exotización, desde una perspectiva que entiende su conocimiento como esotérico, como una construcción con y de lo sobrenatural, noción que como afirma Descola (2012, p. 136) a partir de Durkheim presupone un orden natural determinado por las leyes de ese mundo externo y objetivo, contraponiéndose lo sobrenatural como la incapacidad de acceder a ese orden. Esta jerarquización del conocimiento y la ubicación del pensamiento Kogui en ese orden, elimina la posibilidad de establecer un dialogo horizontal en las negociaciones y articulaciones con instituciones del Estado, de manera que el pensamiento indígena queda relegado a una esfera aparte, "lo cultural", como si fuera un aspecto más y no la centralidad en las negociaciones sobre el territorio ${ }^{13}$.

En varias oportunidades, escuché a líderes indígenas reclamando la representación de su pensamiento como esotérico, como si las relaciones que establecen con los seres de la naturaleza hicieran parte de un pensamiento mágico.

La ontología animista de Descola (2012), parte de que la naturaleza y sus elementos son sujetos en la relación social. Al igual que en el perspectivismo de Viveiros de Castro (2011), se entiende que la humanidad no se refiere a una especie, sino que es una condición de los existentes, que tiene que ver con una interioridad común. Se refiere a

\begin{abstract}
intencionalidad, subjetividad, reflexividad, afectos, aptitud para soñar y significar- así como a características más abstractas aún, como la idea de que comparto con otros una misma esencia o un mismo origen, o que pertenecemos a una misma categoría ontológica (DESCOLA, 2005, p. 103).
\end{abstract}

Por otro lado, el concepto de cosmopolitica de Isabel Stengers resulta bastante útil para el análisis propuesto. Stengers (2003), realiza una crítica a las ciencias occidentales cuestionando su pretendida

\footnotetext{
${ }^{13}$ Es el caso por ejemplo de los ejercicios de ordenamiento ambiental, en los que si bien según la resolución 621 de 2002, las entidades ambientales deben tener en cuenta la visión del territorio indígena en los planes de ordenamiento que afecten su territorio, esta visión no se ha establecido como eje central, por lo contrario se articulan solo prácticas culturales aisladas a lineamientos centrales de las prácticas de conservación occidentales. Por ejemplo, puede que se apoyen los rituales de pagamento, pero no se realiza un ordenamiento a partir de los principios de Sé nenulang.
}

Espaço Ameríndio, Porto Alegre, v. 8, n. 1, p. 135-161, jan./jun. 2014. 
universalidad, y su establecimiento como única verdad que deslegitima otro tipo de conocimientos al categorizarlos como irracionales 0 supersticiosos. La separación de dominios de conocimiento -social y natural-, está en la base de la racionalidad moderna, y soporta esa jerarquización que invalida otros sistemas de conocimiento que plantean otro tipo de relaciones entre los existentes y otros procesos de creación de conocimiento. Es decir, que otras ontologías no podrían constituirse como fuente de conocimiento. Stengers propone el concepto de cosmopolítica para ultrapasar esa lógica del conocimiento, su estructura jerárquica y sus oposiciones binarias. La cosmopolitica sería una política del cosmos, una manera particular de organizar los existentes y de gestionar la alteridad, a partir de un ensanchamiento ontológico que incluye otros seres no humanos (PICAS, 2010). Permite reconocer otros mundos, cuya organización particular puede determinar un sistema epistemológico. En este sistema no acontecen las divisiones binarias entre naturaleza-cultura, sujeto-objeto, físico-espiritual, etc., con los contenidos clásicos de la racionalidad euro-centrada.

El universo Kogui es una ontología animista (DESCOLA, 2012) que ubica en el mismo plano de relacionamiento a los existentes, reconociendo que se comparte un origen y la condición de ser gente con animales, piedras y diversos tipos de entidades humanos y no humanos. En este marco, la Ley de Sé no es simplemente un conjunto de prácticas simbólicas basadas en una construcción relativa, espiritual del mundo. Constituye una cosmopolitica en el sentido de Stengers, como una política particular que establece un orden y una manera en la que los existentes, que teniendo formas diferentes comparten una misma ontología, se relacionan entre si conformando el cosmos, de acuerdo a una política de alteridad que incluye otro tipo de seres no humanos en el continuo social, y con capacidad de actuar y componer un mundo en el que lo material no está separado de lo espiritual; sistema este capaz de generar una epistemología diferente.

Retomemos los jaba y jate, que son el eje central de la Ley de Sé. Como padre y madre, son el origen de algo que deben regular $y$ proteger, asegurándose que cumpla su función en el orden del universo. Cada jaba y jate está inscrito en el territorio en un lugar determinado, de ahí que su traducción al español, en el marco de la lucha por la 
defensa del territorio, sea la de "sitio sagrado". Sin embargo, no se trata de un punto específico, sino de la interrelación de seres y energías de esos espacios, que requieren un manejo especial.

Como había mencionado anteriormente, cada dueño o jaba y jate posee un sewá, que es conocimiento específico del cual depende la función que tiene en el universo, como actúa en este, tanto el como lo que contiene. Por ejemplo, Shikaka, que está ubicada en la desembocadura del rio Ranchería, es la madre de todos los materiales del mar que se usan para pagamento (OGT, 2012, p. 19). Esta jaba (madre) es todas las relaciones de seres que hay en esa desembocadura, además de ser el origen espiritual de los objetos de pagamentos, contiene el Sewá (conocimiento) para usarlos, al que el mama puede acceder en Aluna. En este sentido, Shikaka es la dueña no de una posesión determinada y cerrada, sino de un "dominio" dinámico (FAUSTO, 2008). Es importante resaltar esta condición de dinamismo para no pensar que se trata de jurisdicciones cerradas y predeterminadas del conocimiento y la acción. Como advierte Fausto, un dominio puede apropiarse de otros, ser apropiado, o transformarse respondiendo tanto a las relaciones entre colectivos, como a los procesos de composición de persona. Puede decirse sobre la Sierra Nevada lo que afirma Fausto sobre la mitología amerindia: "implica um mundo atravessado por relações de dominio, mas não uma cartografia cósmica de propiedades distintas e exclusivas. Essas relações de domínio são múltiplas e potencialmente infinitas" (FAUSTO, 2008, p. 341).

La vitalidad de esta jaba, depende no solo de la conservación de este espacio, sino de que sea alimentada espiritualmente y mantenga sus conexiones con las otras entidades del territorio, manteniendo el tejido universal, mediante los flujos de la naturaleza y los pagamentos. Es así como por ejemplo los dueños de las cumbres nevadas deben conectarse con los dueños de las orillas del mar, para lo cual los mamos deben llevar conchas del mar a los nevados y elementos del nevado a las orillas del mar; así mismo los flujos del agua desde los picos nevados descienden a lagunas, aguas subterráneas y ríos hasta llegar al mar y luego regresan a las cumbres con la lluvia (OGT, 2012, p. 17). Todo el territorio debe mantener esa red de conexiones entre jabas 
y jates, por la cual se mantienen los flujos y la circulación de energía y pensamiento que mantiene viva a la Sierra.

Claramente los pagamentos mantienen el tejido de relaciones que componen el cuerpo de la Sierra, pero no se trata solo de un intercambio entre entidades en una relación- un pago por un bien recibido- sino de una composición compleja entre seres con agencia mamo, jaba o jate, y materiales de pagamento- que actúan sobre el mundo, alejando o sanando enfermedades, previniendo la escases y permitiendo la vida mediante el cumplimiento de su función. A partir de esta perspectiva, los materiales de pagamento son primordiales no solo porque permiten un relacionamiento entre entidades, sino por que actúan sobre estas y sobre el mundo.

Los materiales tienen su propio sewá, del cual depende a qué jaba o jate deban ser entregados y con qué fin. Adicionalmente, reciben el sewá que el mamo les entrega en Aluna, transmitiendo su pensamiento o intención al objeto mientras respiran con él. Así mismo, el mamo recibe el sewá del material (URIBE, 1998). Estos sewás del mamo y del material, se conectan con el jaba o jate correspondiente y permiten que este ejerza su función de vigilar, alimentar o sanar.

En su trabajo sobre los Mbyá-guarani, Baptista da Silva (2013) señala la agencia de los objetos a partir de dos aspectos. Hay objetos que son centrales en la constitución de cuerpos y personas, pues su incorporación que se da mediante ingesta, inhalación o cercanía con el cuerpo, permite que ejerzan una función de protección y limpieza, a la vez que pueden ser materializadores de relaciones con otras entidades, "memorias de encuentros pasados" (BAPTISTA DA SILVA, 2013, p. 48) entre entidades que son fluidas y compuestas. Estos dos aspectos pueden aportar para entender la agencia de los materiales de pagamento de Sé nenulang.

Los materiales de pagamento son de diversos tipos: algodón, insectos, plumas, conchas de mar, cuarzos, oro, turmas- que son piedras pequeñas, como cuentas de collar, que fueron talladas por los Tayrona y por lo tanto son consideradas objetos muy poderosos pues contienen el pensamiento de los antiguos. Cada uno tiene un uso específico, pueden ser jefes, vigilantes, alimento, depende de su sewá y de la función que en concordancia el mamo haga que lo constituya. 
Todos los objetos, son gente en aluna y desde ahí tienen determinada su función, su manera de incidir en el mundo. Los objetos de cuarzo por ejemplo, suelen tener la función de vigilar y purificar. La incorporación de estos materiales al cuerpo de la madre permite sanarla de enfermedades, dejarle a ella lo que es suyo, para que después ella no cobre con enfermedades, fenómenos naturales o escases. Los objetos actúan vigilando que las entidades y las personas cumplan con los códigos de la ley de sé.

Así mismo, estos objetos también pueden ser usados en la configuración de persona. En los trabajos espirituales de limpieza o de iniciación de un trabajo por ejemplo, la persona recibe un cuarzo que debe guardar y mantener consigo, pero donde no sea visible, pues se trata de relacionamientos personales e íntimos con la madre. El cuarzo ayuda a limpiar las intenciones de la persona y a que estas, como fueron comunicadas y confiadas a la madre durante el trabajo espiritual, se mantengan y puedan cumplirse. Todo trabajo a realizarse debe quedar registrado en pensamiento para la madre, y un objeto puede acompañar el buen desarrollo del trabajo saneado. Los trabajos de aseguranzas son muy comunes entre los indígenas de la sierra y pueden hacerse por ejemplo para proteger a las personas que realizan algún trabajo en el territorio, o que van a emprender algún viaje. Después de un confieso, en el que se habla con el mamo en un jaba o jate, un lugar sagrado, determinado por este, $\mathrm{y}$ acorde al objetivo del trabajo espiritual, el mamo limpia los pensamientos condensados en algodones que el confesante sostiene en las manos y luego entrega al mamo, quien posteriormente amarra una cuerda en las muñecas del confesante para asegurar su intención y pensamiento. En estos casos los objetos son materializaciones de una relación que se estableció con la madre y un jaba o jate determinado, que al incorporarse al cuerpo de la persona mantienen vivo ese relacionamiento y actúan bien sea protegiendo o limpiando. Al igual sucede con los objetos al incorporarse al cuerpo de la madre, el territorio. Además de establecer las conexiones entre entidades, son incorporados ejerciendo una función específica acorde al dominio de las entidades que relacione y a su propio sewá. Procesos de composición que son infinitos y que deben ser renovados constantemente. 
Sin embargo, hay una característica de estas relaciones que debe ser pensada y que dejo para un análisis posterior, y es sobre las posibles relaciones asimétricas entre estas entidades. Teniendo en cuenta que hay sewás más poderosos, que establecen dominios dinámicos, ¿de qué manera pesa esto en este tejido y en la apropiación de fluidos entre entidades (materiales, mamo, dueño, territorio)?, ¿Cómo operan las macro relaciones entre colectivos y la micro producción de personas, en estas apropiaciones?

Los objetos de oro, así como los que fueron tallados por los Tayrona, han sido removidos de su lugar por los guaqueros (saqueadores de objetos arqueológicos), para ser vendidos a coleccionistas privados (lo cual es ilegal de acuerdo a la legislación colombiana), o bien son entregados a arqueólogos, o extraídos por ellos mismos para ser estudiados y exhibidos en museos. Estas acciones están soportadas tanto en la autoridad de la arqueología como un saber experto (que desde su visión deslegitima las prácticas de guaqueros, si bien el efecto en las dinámicas sociales locales es el mismo) y en el discurso del patrimonio, planteando un conflicto entre los significados de los objetos para los pueblos actuales que hacen uso de ellos, y su relación con estos discursos globales dominantes.

\section{Dominios epistemológicos diferentes}

Si bien los Kogui fortalecen su identidad reiterando la importancia de la tradición y del modo de vida de los antiguos, alimentando ese ideal detenido en el tiempo, no constituyen una sociedad aislada ni estática (URIBE, 1998). Su cosmopolitica es capaz de abrazar la diferencia, logrando enfrentar los devenires del contacto con otras sociedades, que no se entienden como ajenas a la propia. Uribe (1998) argumenta como el orden del mundo Kogui, en el que ellos se reconocen como hermanos mayores, involucra a otras sociedades, a quienes reconocen como hermanos menores, sistema que se ha desarrollado a lo largo de una historia de contactos entre sociedades. Los hermanitos menores o bunachi, somos al igual que ellos, hijos de la misma madre, y para usar los términos usados en este análisis, estamos 
incluidos en su cosmopolitica, tenemos un lugar en la Ley de Sé. Solo que no es una ubicación mecánica, no tenemos asignado directamente un jaba y jate para lo que pertenece a los hermanos menores. Entidades y elementos provenientes de otras sociedades tienen un sentido en su cosmopolitica en tanto es allí donde originalmente fue concebido todo lo que existe. Si bien puede hablarse de indigenización de elementos de afuera (BOCAREJO, 2002) hay que tener precauciones para no caer en entender estos procesos como mecanismos predeterminados, cerrados, o que a priori delimitan qué es lo Kogui y qué es lo ajeno. Una de las cuestiones más interesantes de estos procesos, para el caso Kogui, es que esas barreras entre lo propio y lo ajeno se borran sin que se diluya aquí la diferencia. El sentido que una entidad tenga dentro de su universo, no impide ver que pueda tener otros sentidos para otras sociedades. Lo que quiero decir es que la manera como entidades diferentes puedan relacionarse en la cosmopolitica Kogui, no predetermina un lugar ni una función en su orden, si no que esta se da a partir de dinámicas puntuales históricas de relaciones entre colectivos, así como de microprocesos de la formación de persona. Estamos hablando de nuevo de dominios y de su capacidad de configurarse y reconfigurarse a partir de procesos de relacionamiento social y composición de persona. Voy a mencionar dos ejemplos para intentar clarificar este postulado.

Lo que diferencia a los hermanos mayores de la sierra de los hermanitos menores, es que siendo hijos de la misma madre, haciendo parte de la misma familia universal, los hermanitos menores no recibieron el conocimiento de la madre, no recibieron sewá. Por lo tanto no tienen la sabiduría espiritual que se requiere para manejar el territorio, y en su desconocimiento muchas veces son irrespetuosos con la madre (ZHIGONESHI, 2009), sin embargo, los hermanitos menores tienen su propio conocimiento y sus propias prácticas y dominios. La manera como ese conocimiento de bunachi tiene sentido en el territorio de la sierra depende de cómo se da el contacto, de la experiencia personal y la relación macro entre colectivos.

El colectivo audiovisual Zhigoneshi, está conformado por indígenas Wiwa, Kogui y Arhuacos. Se creó con el objetivo de autorepresentarse y como estrategia para hacer visibles los problemas 
que sufren sus comunidades, dar a conocer parte de su pensamiento para que los bunachi entiendan la importancia de que los hermanos mayores puedan cumplir su misión de cuidar la Sierra. Las actividades de este colectivo tenían que tener la aprobación de los mamos, quienes se sentían un poco incómodos ante las cámaras, invadidos. No fue sino hasta que uno de ellos identificó quien era el jate de las cámaras, que se hizo el respectivo pagamento y los mamos hicieron trabajo tradicional para que ese jate, Sey Arimaku, el dueño de la oscuridad, autorizara que las cámaras y los equipos pudieran transmitir fielmente la palabra indígena (ZHIGONESHI, 2012).

La tecnología en esta cosmopolitica es otra entidad que hace parte de un dominio. El punto está en que este dominio del jate de la oscuridad se apropió de esta otra entidad constituyéndose en su origen regulador, en un momento en el que es necesario fortalecer alianzas con los hermanitos menores. El hecho de que sea legitimado para poder comunicar el pensamiento de la sierra, no implica que deje de tener otros usos por parte de otras personas.

En todo caso, si bien la cosmopolitica Kogui es un sistema abierto a la diferencia no quiere decir que sea ausente de conflictos.

Los pueblos de la Sierra nunca se han mantenido en aislamiento y por el contrario, han intentado mantener una interlocución con el Estado colombiano para dialogar sobre sus problemas y las intromisiones de los hermanitos menores. Enmarcados en conflictos diversos por la expansión colonialista de campesinos en su territorio, el avance de cultivos de marihuana y coca con fines ilegales, el conflicto armado, intereses turísticos, de megaproyectos, así como de conservación medioambiental y cultural, los pueblos de la Sierra comenzaron en la década de 1970 un proceso organizativo para luchar por la defensa de su territorio y su autonomía. Esto implico la creación de instancias y de autoridades que antes no existían y que acarrearon en su momento un desequilibrio mientras reorganizaban el lugar de las nuevas autoridades en relación a las autoridades tradicionales, definiendo dominios y canales de comunicación ${ }^{14}$.

\footnotetext{
${ }^{14}$ En todo caso vale la pena mencionar que esto no está exento de conflicto y que naturalmente hay posturas divergentes en cuanto al relacionamiento hacia afuera dentro de las organizaciones y en las comunidades. Si bien las organizaciones cuentan con representatividad y credibilidad de los pueblos
}

Espaço Ameríndio, Porto Alegre, v. 8, n. 1, p. 135-161, jan./jun. 2014. 
En este marco, han intentado reivindicar y defender su visión del territorio, intentado que esta prime en políticas y acciones que afectan su territorio ancestral. Las dificultades alrededor de los objetos y materiales de pagamento son un claro ejemplo de encuentro de nociones y perspectivas diferentes en conflicto.

Como se mencionó inicialmente, el territorio actual de la Sierra Nevada de Santa Marta fue habitado por los Tayrona, de quienes los grupos indígenas actuales reclaman ser descendientes. Los Tayrona son reconocidos por las ruinas arqueológicas de una ciudad que se conoce como Teyuna o ciudad perdida, así como por sus valiosos objetos tallados en piedras, cerámica y oro que han sido encontrados en el territorio.

La actividad de la guaqueria, como se conoce localmente a los saqueos de "tesoros indígenas" se fortaleció en la Sierra a partir de 1970, conformándose una mafia fortalecida desde contactos en Bogotá, que manejaban las redes para la venta de las piezas encontradas (VALDERRAMA y FONSECA, 1981). Los rumores de que los guaqueros habían encontrado las ruinas de una gran ciudad indígena llegaron al ICAN (Instituto Colombiano de Antropología), quienes enviaron una expedición para asegurarse de que era cierto el hallazgo y posteriormente tomar medidas para regular su acceso y controlarlo, basándose en que se trataba de patrimonio nacional. Posteriormente, se declara el área como Parque Arqueológico Teyuna ciudad perdida, bajo la administración del ICAN. Sin embargo, para las actividades de rescate y restauración se contó con la ayuda de guaqueros (VALDERRAMA y FONSECA, 1981). Incluso, lo que se dice en el área es que las actividades arqueológicas que se han desarrollado y se siguen desarrollando en la zona, se hacen con el apoyo de guaqueros, hasta el punto que algunas personas de la zona llaman a los arqueólogos guaqueros profesionales.

En este marco, los pueblos indígenas han visto como objetos que para unos son entendidos como "guacas" o "tesoros" y para otros "objetos arqueológicos", y que para ellos son materiales u objetos sagrados, ahora son escasos en el territorio. Ahora se encuentran en

indígenas, no puede considerarse que constituyan un bloque homogéneo. Para profundizar en estos aspectos organizativos y de interlocución con el Estado, ver (ULLOA, 2004).

Espaço Ameríndio, Porto Alegre, v. 8, n. 1, p. 135-161, jan./jun. 2014. 
museos y colecciones privadas, lejos de su origen, desconectados y $\sin$ alimento, lo que causa problemas en el territorio.

Esta situación confronta no solamente valores simbólicos sino discursos pertenecientes a epistemologías diferentes en un orden jerárquico. La arqueología y los museos se basan en el discurso del patrimonio que sobrepone intereses globales ante intereses o saberes locales. Legitima el sacar los objetos del territorio bajo el argumento de que constituyen símbolos de la identidad y memoria nacional, mientras que los valores y sentidos propios locales son, en el mismo sentido de Reichel comentado anteriormente, representaciones simbólicas, que no se sustentan en un sistema de conocimiento como si lo hace el patrimonio, sustentado en la arqueología y la construcción de nación. Como afirma Clifford (1999), la lógica del patrimonio y los museos nacionales es que los objetos respondan a un sentido cosmopolita entendido este término en su sentido clásico como ser habitante del universo sin estar atado a localidades- los objetos deben trascender sus sentidos locales para ser un símbolo de la nación; los sentidos locales pasan a segundo plano para dar paso a sentidos más globales, donde la nación pueda apropiarlos y dibujar su identidad.

Sin embargo no se puede asumir que el patrimonio es un bloque homogéneo. Es un discurso que está sujeto a transformaciones acordes al contexto histórico. Es posible que ciertas corrientes estén buscando como darle peso a los sentidos locales en la arena global.

Ahora los pueblos de la Sierra están acudiendo a los procesos de patrimonialización para proteger sus objetos y por ende su territorio; quieren usar ahora el mismo discurso que sacó sus objetos del territorio, para pedir que regresen a su lugar de origen y se construya una estrategia de protección de estos y los lugares sagrados, jaba y jate.

En este marco, los pueblos de la Sierra se han acercado a algunos museos que tienen objetos provenientes de su territorio. Dos de estas visitas están registradas en el documental de Zhigoneshi "Resistencia en la Línea Negra" (2011). En este, se ve como los mamos en sus visitas manifestaban con tristeza como esos jefes y vigilantes estaban lejos de su origen, como castigados y sin poder cuidar de la tierra como es su misión. Preocupados por ver como la tierra se ha debilitado por el hecho de que ya no están sus guardianes donde deben estar, permitiendo el 
flujo de fuerza y energía. En la visita al Museo del Oro de Bogotá, la funcionaria que los recibió, exaltó la labor del museo como entidad que recuperó los objetos y no permitió que fueran vendidos en el exterior, y que hace posible que los objetos sean apreciados por todos los colombianos a quienes pertenecen; a su vez escuchó con desdén las apreciaciones de los mamos sobre la tristeza que les da ver a esos jefes "como presos, sin alimento", y guardó silencio sobre su plan de recuperarlos y llevarlos de vuelta a su territorio. Por otro lado, en la visita al Museo Nacional, también en Bogotá, los funcionarios del Museo que los recibieron los acompañaron y escucharon con respeto, $y$ manifestaron su interés en analizar cómo podría hacerse una reparación, y establecer un diálogo para que los mamos decidan qué objetos pueden mostrarse y cuáles no, y cuáles deberían volver a su territorio, si es que esto es posible.

Sin embargo, no es una cuestión fácil, ni desde la esfera jurídica ni desde la visión indígena. Estos materiales sagrados han sido retirados de su origen determinado y solo después de un buen trabajo espiritual podrían llevarse donde pertenecen, es decir a su jaba o jate correspondiente. No pueden ubicarse en cualquier lugar porque así no podrían cumplir su misión, es necesario conocer además cuál es su sewá. Adicionalmente, por haber salido del territorio y haber pasado por otras redes de flujos, por estar alejados de las conexiones de la Sierra y estar aislados de ese alimento espiritual, así como por estar expuesto a miradas y pensamientos de muchas personas, estos materiales están sucios y pueden ser peligrosos, han sido afectados por otras entidades. Algunos tienen un sewá muy poderoso que podría causar estragos sino es limpiado, equilibrado y puesto en el lugar al que pertenece.

Los 4 pueblos de la Sierra acudieron al Ministerio de Cultura para buscar la declaración de los objetos, los sitios sagrados y su conocimiento asociado como Patrimonio Cultural material e inmaterial de la nación. Para ellos tiene sentido en tanto pueda funcionar como una figura de protección, sobre la cual se puede construir un plan de salvaguardia sobre lo que ellos consideren es una amenaza o riesgo. En Colombia son las mismas poblaciones las que deben realizar la postulación del "bien" a declarar, reunir los argumentos por los que debe declararse como patrimonio, y elaborar el plan de salvaguarda. Es 
un proceso que hasta ahora está comenzando y que implica un reto enorme para ambos lados; los mamos y las organizaciones indígenas no están dispuestos a dejar pasar la integralidad de lo material y el pensamiento que confluye en los objetos sagrados y en el territorio. Sería un proceso pionero: para el Ministerio representa el reto de manejar un "hibrido" entre algo tangible y algo intangible, como categorías básicas reconocidas para el patrimonio desde los lineamientos de la UNESCO que separa lo material de lo inmaterial. La intención de abordar el caso de la Sierra como un bien "integral" es un proceso interesante, que puede dar cuenta de una transformación de las prácticas de patrimonio. Habrá que ver como se efectúa el juego entre los sentidos y valores globales y locales, y si realmente las prácticas asociadas al patrimonio pueden transformarse y responder a las expectativas indígenas que han encontrado en ella una posibilidad de protección, desde su cosmopolitica. En palabras de Escobar (2005) ¿será posible que el conocimiento local incida y reconfigure el discurso de lo global estableciendo otros relacionamientos?.

Para los pueblos de la Sierra representa el reto también de entender plenamente las implicaciones de tal proceso, partiendo de las connotaciones que tienen las palabras y las estrategias en otro sistema de conocimiento y la manera como es interpretada su "cultura," en un marco de dinámicas coloniales que no solo no reconoce su epistemología como tal, sino que también tiende a absorber y neutralizar la diferencia al entender, detrás de esa noción de cultura, algo estático, que debe ser descrito en sus términos actuales para evitar que se transforme. Esos juegos y articulaciones de dominios de conocimiento y poder son lo interesante de este proceso que apenas comienza.

\section{El lugar del antropólogo/a}

Siendo antropólogos, ¿cómo nos ubicamos en este panorama? La primera vez que fui a un recorrido para hacer pagamentos con los Kogui y Arhuacos hacia parte del equipo del Parque Nacional Natural Sierra 
Nevada (PNN SNSM). El viaje era a las lagunas sagradas del páramo ${ }^{15}$ de rio Frio, por encima de los 3500 msnm. Mi participación en la expedición se decidió a última hora y no hubo tiempo de avisar quien era yo ni a que iba. Los mamos no me conocían. En la actividad de confieso previa a iniciar el viaje, no hablé de por qué iba, si no de la actitud de respeto hacia el lugar que sentía. Una vez en el viaje, interactuamos con los mamos, pero inicialmente no hubo oportunidad de contar cual era mi trabajo en el Parque ni por qué estaba acompañándolos. Solo sabían que yo había asumido la responsabilidad de registrar en video los pagamentos, pues el indígena que iba a hacer esa tarea había decidido no ir, si bien esta no era la razón central de mi presencia en el viaje. Después de 2 días de camino, llovía muy fuerte y no había buena visibilidad, nos perdimos. Esa noche acampamos los 5 que éramos del equipo del parque sin saber dónde estaba otro compañero nuestro ni los mamos. Al medio día siguiente, ellos nos encontraron. Ya habían realizado los pagamentos y se mostraban reacios a hablar conmigo sobre los trabajos hechos. El compañero que se había quedado con ellos nos contó que le decían que no se preocupara por nosotros, que la lluvia y la neblina habían caído por algo, la madre tal vez no quería que fuera tanta gente a los pagamentos; a las lagunas no les gusta tanto forastero. Al terminar la primera jornada de regreso, les pedí a los mamos hablar con ellos. Hablaban en lengua. Si bien yo no hablo Kogui, entendía que repetían munji, que quiere decir mujer. Una vez me dieron la palabra, supe que tenía que legitimar mi presencia en un espacio de hombres, contarles sobre mi experiencia con otros grupos indígenas, y sobre los objetivos de mi trabajo, siendo consiente que esos objetivos me hacían estar en lugares en los que no es usual la presencia de mujeres. Ellos se reían y me explicaron que primero habían pensado que como era mujer, sería la cocinera. Como no cociné, pensaron que era turista, y los turistas no tienen por qué ir a las lagunas, mucho menos grabar; que ahora entendían por qué estaba allá, y que ahora si querían hacer el video, hablando de la importancia de los pagamentos que habían hecho.

Aparte de las reflexiones sobre el hecho de ser antropóloga mujer

\footnotetext{
${ }^{15}$ Páramo es un ecosistema de montaña que tiene lugar entre los 3000 y los 5000 msnm, que por sus características suele ser reservorio y productor de fuentes hídricas.
}

Espaço Ameríndio, Porto Alegre, v. 8, n. 1, p. 135-161, jan./jun. 2014. 
en estos espacios, tema que aún estoy pensando y guardo para posteriores análisis, fue evidente la imposibilidad de relación con los mamos y las jaba y los jate del páramo, si no era claro para ellos cual era mi función, cual era mi ubicación en el orden. No lo había mencionado en el confieso, que era el momento para que los mamos y la madre lo supieran, era el momento para haber registrado mi función y haber actualizado la relación con las entidades no humanas, a la vez que establecía desde que dominio me situaba y a partir de esto, que posibilidades de relación tendría con las entidades sagradas que iríamos a visitar.

Actualmente mi investigación de maestría se articula, desde la organización indígena Gonawindua Tayrona OGT, al proceso de patrimonialización de los objetos y territorio de la Sierra Nevada con el Ministerio de Cultura, en cuyo marco mi función es la revisión de los documentos de acuerdos con el Estado y el proceso de relacionamiento con museos. Mi función corresponde al dominio que me es afín, al análisis del conocimiento y los papeles de los hermanitos menores, desde las reflexiones y expectativas locales.

Para interactuar con las entidades no humanas, en este caso, es determinante comunicarles la función, y esto se hace mediante los trabajos espirituales, como uno de los elementos que actualiza el estatus ontológico de jabas, jates, materiales sagrados y el propio. En este marco, es importante asumir que estos procesos llevan implícita la composición de la persona y por lo tanto algunos son cuestiones privadas que no se deben contar, así constituyan "joyas" del conocimiento etnográfico que quisiéramos comunicar. Es la función desde la que se actúa la que define qué puede ser contado y qué no, así como los dominios a los que se puede acceder.

La interacción con entidades que Ilamaríamos de "afuera", desde el mundo indígena, son una expansión del mundo atravesado por dominios de flujos y acciones. En estos casos en que se sobreponen epistemologías en la lucha política por el reconocimiento y la defensa del territorio, el mundo indígena claramente debe ajustarse a los términos y exigencias del modelo dominante para ser reconocido en esa lógica, con el riesgo de que la diferencia sea normalizada. Sin embargo, este ejercicio de traducción y de lucha política no surge como respuesta 
pasiva a las exigencias de los discursos globales. Lo que Carneiro da Cunha (2009) Ilama "cultura" entre comillas, como ese discurso creado que instrumentaliza la cultura (sin comillas) para reivindicar derechos no responde a una política interétnica diferente a una política interna, como ella afirma; tampoco se trata de campos separados o discontinuos. Se trata de la extensión de una manera de ordenar la alteridad, de la cosmopolitica que no funciona como sistema cerrado y no es estática, sus dominios y procesos van modificándose y recomponiéndose de acuerdo a los encuentros con alteridades diversas. Se trata entonces no solo de las entidades espirituales del territorio, de las humanas y no humanas, sino también de nuevas entidades que en el marco de las interacciones del sistema global tienen ahora incidencia en la configuración del territorio.

Es innegable, en todo caso, que en ese ejercicio de traducción y de lucha política en el que confluyen entidades con epistemologías diferentes, prima una estructura de poder que se ejerce de muchas formas. Sin embargo, es importante tener en cuenta que estos procesos no implican sujetos o receptores pasivos, se trata de un encuentro en el que se reconfiguran y se recomponen las entidades en relación, y que en estos procesos cabe la posibilidad que los sentidos dominantes puedan ser subvertidos por conocimientos locales, para lo cual es necesario hacer evidente el flujo continuo entre estas escalas pretendidamente excluyentes (local-global).

La perspectiva cosmopolítica me parece que es una invitación a posibilitar que se tejan relaciones en múltiples escalas y localidades. Como afirma Stengers (2003), lo interesante de las relaciones entre sistemas de conocimiento diversos es entender sus diferencias en sus procesos de construcción, e intentado relacionarlos desde una posición de simétria, pero también haciendo evidentes los marcos que establecen jerarquías y los mecanismos por medio de los cuales se ejerce el poder y se tiende a absorber la diferencia. En este sentido, y en el marco de la cosmopolitica kogui, la función del antropólogo no es ya servir de intermediario entre redes epistemológicas diferentes, ni ser traductor; el relacionamiento se da entre autoridades, los indígenas hablan por sí mismos y son completamente conscientes de las limitaciones de la 
traducción, en ambos sentidos16. Dejando atrás los momentos coloniales en que el antropólogo era el protagonista, considero que nuestra función puede construirse a partir de un acompañamiento en el tejido de las relaciones de entidades de redes diversas que configuran el territorio, a partir de aportar elementos para el análisis crítico de la diferencia que permita reivindicar conocimientos locales, entender las diferencias en los sistemas de conocimiento, y los mecanismos mediante los cuales se ejercen jerarquizaciones, teniendo en cuenta que se trata de dominios no totalitarios, no homogéneos y en constante transformación.

En adelante hay infinidad de preguntas sobre el proceso que comienza, sobre las condiciones que han permitido que se de esta articulación de epistemologías y las transformaciones y expectativas de cada una; sobre la posibilidad de una figura de protección que no encapsule la diferencia y le permita seguir sus fluidos y expansión de dominios, con pleno conocimiento de las posibles implicaciones políticas; y la posibilidad de que esta figura incida en otros campos que los hermanitos menores han separado, pero que hacen parte de la integralidad de Sé nenulang.

\section{Bibliografia}

BAPTISTA DA SILVA, Sergio. Cosmo-ontológica mbyá-guarani: discutindo o estatuto de "objetos" e "recursos naturais". Revista de Arqueologia, São Paulo, v. 26, n. 1, p. 42-54, 2013.

BOCAREJO, Diana. Indigenizando "lo blanco": conversaciones con Arhuacos y Koguis de la Sierra Nevada de Santa Marta. Revista de Antropología y Arqueología, Bogotá, v. 13, p. 3-44, 2002.

CARNEIRO DA CUNHA, Manuela. Cultura com aspas. São Paulo: Cosac Naify, 2009.

CLIFFORD, James. Itinerarios Transculturales. Barcelona: Gedisa, 1999 [1997].

DESCOLA, Philippe. Construyendo naturalezas, ecologia simbólica y práctica social.

${ }^{16}$ Ver por ejemplo Mestre (2013).

Espaço Ameríndio, Porto Alegre, v. 8, n. 1, p. 135-161, jan./jun. 2014. 
In: DESCOLA, Philippe; PÁLSSON, Gisli (Org.). Naturaleza y sociedad, perspectivas antropológicas. Cidade do México: Siglo XXI editores, 2001 [1996]. p. 101-123.

DESCOLA, Philippe. Mas allá de la naturaleza y la cultura. Revista Etnografias Contemporáneas, Buenos Aires, v. 1, n. 1, p. 75-96, 2005.

Más allá de naturaleza y cultura. Buenos Aires: Amorrortu, 2012.

ESCOBAR, Arturo. El lugar de la naturaleza y la naturaleza del lugar: ¿globalización o postdesarrollo?. In: LANDER, Edgardo (Org.). La colonialidad del saber: eurocentrismo y ciencias sociales, perspectivas latinoamericanas. Buenos Aires: CLACSO, 2005. p. 68-87.

FAUSTO, Carlos. Donos demais: maestria e domínio na Amazônia. Mana, Rio de Janeiro, v. 14, n. 2, p. 329-266, 2008.

GIRALDO, Natalia. Camino en espiral. Territorio sagrado y autoridades tradicionales en la comunidad indígena iku de la sierra nevada de Santa Marta, Colombia. Revista Pueblos y fronteras digital, Cidade do México, v. 6, n. 9, n. p., 2010. Disponível em: http://www.pueblosyfronteras.unam.mx/a10n9/art_07.html . Acesso em: 28 jun. 2014.

INGOLD, Tim. The perception of environment: essays in livelihood, dwelling and skill. New York: Routledge, 2001.

Trazendo as coisas de volta à vida: emaranhados criativos num mundo de materiais. Horizontes Antropológicos, Porto Alegre, v. 18, n. 37, p. 25-44, 2012.

LATOUR, Bruno. Nunca fuimos modernos. Cidade do México: Siglo XXI editores, 2012.

MARCUS, George. Etnografía en/del sistema mundo: el surgimiento de la etnografía multilocal. Alteridades, Cidade do México, v. 11, n. 22, p. 111-127, 2001. Disponível em: http://www.redalyc.org/articulo.oa?id=74702209 . Acesso em: 20 out. 2013.

MESTRE, Yanelia. El conflicto subyacente en el uso de la lengua castellana: proceso de acercamiento a la sociedad mayoritaria y distanciamiento del conocimiento original. Revista Zhigoneshi, Santa Marta, v. 15, n. 13, 2013.

OGT. Jaba y jate: espacios sagrados del território ancestral Sierra Nevada de Santa Marta. Santa Marta: OGT, 2012.

ORRANTIA, Juan Carlos. Matices Kogui. Representaciones y negociación en la marginalidad”. Revista Colombiana de Antropología, Bogotá, v. 38, p. 45-75, 2002.

PICAS, Joan. "Cosmopolítica como "cosmoética": del universalismo occidental a las políticas de un mundo común. ISEGORIA, Madrid, n. 42, p. 55-72, 2010. 
REICHEL, Gerardo. Training for the priesthood among the Kogui of Colombia. In: WILBERT, J. (Org.). Enculturation in Latin America. LosAngeles: University of California, 1976. p. 265-288.

Los Kogui. Bogota: Procultura, 1985. Tomo I y II.

REICHEL DOLMATOFF, Gerardo. Los Kogi: una tribu de la Sierra Nevada de Santa Marta, Colombia. Bogotá: PROCULTURA/Nueva Biblioteca Colombiana/Editorial Presencia, 1985. 2 vols.

SAHLINS, Marshall. O pessimismo sentimental e a experiência etnográfica: parte I. Mana, Rio de Janeiro, v. 3, n. 1, p. 41-73, 1997a.

O pessimismo sentimental e a experiência etnográfica: parte II. Mana, Rio de Janeiro, v. 3, n. 2, p. 103-150, 1997.

Dos o tres cosas que sé acerca del concepto de cultura. Revista Colombiana de Antropología, Bogota, v. 37, p. 290-327, 2001.

STENGERS, Isabel. Cosmopolitiques II. París: Éditions La Découvert, 2003.

ULLOA, Astrid. La construcción del nativo ecológico: complejidades, paradojas y dilemas de la relación entre los movimientos indígenas y el ambientalismo en Colombia. Bogotá: ICANH, 2004.

URIBE, Carlos Alberto. De la Sierra Nevada de Santa Marta, sus ecosistemas, indígenas y antropólogos. Revista de Antropología, Bogotá, v. 4, n. 1, p. 5-36, 1988

De la vitalidad de nuestros hermanos mayores de la nevada. Revista de Antropología y arqueología, Bogotá, v. 10, n. 2, p. 9-92, 1998.

VALDERRAMA, Bernardo; FONSECA, Guillermo. Exploraciones en la vertiente norte de la sierra nevada de santa marta. Boletin - Museo Del Oro Banco De La Repùblica, Bogotá, v. 4, p. 1-41, 1981.

VIVEIROS DE CASTRO, Eduardo. A inconstância da alma selvagem: e outros ensaios de antropologia. São Paulo: Cosac Naify, 2011.

\section{Otros recursos}

Documentales audiovisuales:

Zhigoneshi. "Palabras mayores" I y II. 2009.

. "Resistencia en la línea negra". 2011.

Espaço Ameríndio, Porto Alegre, v. 8, n. 1, p. 135-161, jan./jun. 2014. 
ANA MILENA HORTA PRIETO - Tejiendo entre redes diversas

. "Sey Arimaku, la otra oscuridad”. 2012.

Espaço Ameríndio, Porto Alegre, v. 8, n. 1, p. 135-161, jan./jun. 2014. 